\title{
SCHEME INDEPENDENCE AS AN INHERENT REDUNDANCY IN QUANTUM FIELD THEORY
}

\author{
JOSÉ I. LATORRE \\ Departament d'Estructura $i$ Constituents de la Matèria, Universitat de Barcelona, and \\ I.F.A.E., Diagonal 647, E-08028 Barcelona, Spain \\ TIM R. MORRIS \\ Department of Physics, University of Southampton, Highfield, Southampton SO17 1BJ, \\ $U K$

\begin{abstract}
The path integral formulation of Quantum Field Theory implies an infinite set of local, Schwinger-Dyson-like relations. Exact renormalization group equations can be cast as a particular instance of these relations. Furthermore, exact scheme independence is turned into a vector field transformation of the kernel of the exact renormalization group equation under field redefinitions.
\end{abstract}

Relations for the Green functions of a quantum field theory are in general traced down to symmetries of the partition function. In particular, the path integral allows for the basic symmetry of field redefinitions. A vast family of Schwinger-Dyson like relations follows by taking the infinitessimal field redefinition $\tilde{\varphi}^{\alpha}=\varphi^{\alpha}-\theta^{\alpha}[\varphi, t]$

$$
\int \mathcal{D} \varphi \partial_{\alpha}\left(\theta^{\alpha} \mathrm{e}^{-S}\right)=0 \quad \Rightarrow\left\langle\partial_{\alpha} \theta^{\alpha}\right\rangle=\left\langle\theta^{\alpha} \partial_{\alpha} S\right\rangle
$$

where we have introduced the compact notation

$$
\partial_{\alpha} \equiv \frac{\delta}{\delta \varphi^{\alpha}}
$$

$\alpha$ standing for momentum and other possible quantum numbers.

There is a deep connection between this observation and the exact renormalization group. Remarkably, the exact renormalization group equation itself, 2 . formulated by Bolchinski, 1 can be written as the integrand of a field redefinition transformation 0. ⿴囗⿱一一 To see this, let us first recall the dimensionless form of Polchinski's equation

$$
\begin{aligned}
\partial_{t} S+d \int_{p} \varphi_{p} \frac{\delta S}{\delta \varphi_{p}}+\int_{p} \varphi_{p} & p^{\mu} \frac{\partial}{\partial p^{\mu}} \frac{\delta S}{\delta \varphi_{p}}= \\
& \int_{p} c^{\prime}\left(p^{2}\right)\left(\frac{\delta S}{\delta \varphi_{p}} \frac{\delta S}{\delta \varphi_{-p}}-\frac{\delta^{2} S}{\delta \varphi_{p} \delta \varphi_{-p}}-2 \frac{p^{2}}{c\left(p^{2}\right)} \varphi_{p} \frac{\delta S}{\delta \varphi_{p}}\right)
\end{aligned}
$$

where $t \equiv \ln \frac{\mu}{\Lambda}, \mu$ being some fixed physical scale and $\Lambda$ the Wilsonian cutoff which is taken towards $0, S[\varphi, t]$ is a functional of $\varphi$ and a function of $t$ and $c\left(p^{2}\right)$ is the regulating function defining classes of schemes.

We can now check that Polchinski's equation is identical to

$$
\partial_{t} \mathrm{e}^{-S}=\partial_{\alpha}\left(\Psi^{\alpha} \mathrm{e}^{-S}\right)
$$


where the functional $\Psi^{\alpha}$ corresponds to

$$
\Psi^{\alpha}[\varphi, t]=(D-d(t)) \varphi_{p}+p^{\mu} \frac{\partial \varphi_{p}}{\partial p^{\mu}}+c^{\prime}\left(p^{2}\right)\left(\frac{\delta S}{\delta \varphi_{-p}}-2 \frac{p^{2}}{c\left(p^{2}\right)} \varphi_{p}\right) .
$$

Two facts are now clear:

- The equation is traced to the field redefinition

$$
\tilde{\varphi}^{\alpha}=\varphi^{\alpha}-\delta t \Psi^{\alpha}[\varphi, t] .
$$

- Upon integration, Polchinski's equation implies

$$
0=\partial_{t} \int \mathcal{D} \varphi \mathrm{e}^{-S}=\int \mathcal{D} \varphi \partial_{\alpha}\left(\Psi^{\alpha} \mathrm{e}^{-S}\right)=0,
$$

that is, the l.h.s. vanishes because low-energy physics does not depend on the cutoff, whereas the r.h.s. is zero because it is the total derivative term emerging from a field redefinition.

The field redefinition symmetry underlying the renormalization group does also control its scheme dependence. It is easy to compute the change of the kernel in the exact renormalization group equation under a field redefinition. Starting from $\Psi^{\alpha}$ and redefining $\varphi^{\alpha} \rightarrow \varphi^{\alpha}-\theta^{\alpha}$, produces the change

$$
\begin{aligned}
\delta \Psi^{\alpha}[\varphi, t] & \equiv \hat{\Psi}^{\alpha}[\varphi, t]-\Psi^{\alpha}[\varphi, t] \\
& =\partial_{t} \theta^{\alpha}+\theta^{\beta} \partial_{\beta} \Psi^{\alpha}-\Psi^{\beta} \partial_{\beta} \theta^{\alpha} .
\end{aligned}
$$

This can be further framed as a standard vector field transformation plus the explicit $t$ derivative,

$$
\delta \Psi^{\alpha} \overrightarrow{\partial_{\alpha}}=\partial_{t} \theta^{\alpha} \overrightarrow{\partial_{\alpha}}+\left[\theta^{\beta} \overrightarrow{\partial_{\beta}}, \Psi^{\alpha} \overrightarrow{\partial_{\alpha}}\right]
$$

The above equations suggest a more compact notation. Let us put the differential on the left and define the following shorthand

$$
\theta \equiv \overrightarrow{\partial_{\alpha}} \theta^{\alpha} \quad, \quad A_{t} \equiv \overrightarrow{\partial_{\alpha}} \Psi^{\alpha} \quad, \quad \delta A_{t} \equiv \overrightarrow{\partial_{\alpha}} \delta \Psi^{\alpha} \quad \text { and } \quad D_{t} \equiv \partial_{t}-A_{t}
$$

In this notation the exact RG (任) simply reads

$$
D_{t} \mathrm{e}^{-S}=0 \text {. }
$$

This new form of the exact renormalization group equation deserves a more detailed inspection. Let us in turn discuss its transformation properties and, then, its geometrical interpretation.

By (1), the change in the action under a field redefinition is just

$$
\delta \mathrm{e}^{-S}=\theta \mathrm{e}^{-S}
$$

and, either by integrating by parts the derivative in (9), or directly from (8),

$$
\delta A_{t}=\partial_{t} \theta-\left[A_{t}, \theta\right] \equiv\left[D_{t}, \theta\right] .
$$


Now it is easy to see that our transformation for $\Psi^{\alpha}$ does indeed ensure that the exact RG transforms covariantly:

$$
\begin{aligned}
\delta\left(D_{t} \mathrm{e}^{-S}\right) & =-\delta A_{t} \mathrm{e}^{-S}+D_{t} \delta \mathrm{e}^{-S} \\
& =-\left[D_{t}, \theta\right] \mathrm{e}^{-S}+D_{t} \theta \mathrm{e}^{-S} \\
& =\theta\left(D_{t} \mathrm{e}^{-S}\right) .
\end{aligned}
$$

The functional $A_{t}$ emerges as a connection in the space of actions fibered over renormalization group time. Local field redefinition symmetry is maintained through the presence of $A_{t}$, which is in charge of notifying to neighbouring points what choice of fields has been made. In this fashion the relative weight among field configurations is modified along the flow.

It is natural to ask what are the (observable) invariants associated to the general field redefinition symmetry. These may be local or global. The fact that the renormalization group time is one-dimensional explicitly forbids the existence of the former type: there is no field strength in one dimension. Only integrated invariants can be obtained. Indeed, the Wilson line starting from $t=t_{0}$ is of interest:

$$
\Phi(t)=P \exp \left(-\int_{t_{0}}^{t} d s A_{s}\right),
$$

where $P$ is path ordering, placing as usual the later $A$ s to the right (thus $\partial_{t} \Phi(t)=$ $-\Phi(t) A_{t}$ and $\left.\partial_{t} \Phi^{-1}(t)=-A_{t} \Phi^{-1}(t)\right)$.

Let us put together our line of thought. A particular exact RG equation is characterized by its kernel $\Psi^{\alpha}$. The freedom to choose the form of this kernel is related to field redefinitions. Infinitessimally, two kernels, expressed in terms of the action $S$, are physically equivalent if they are related by

$$
\delta \bar{A}_{t}[\varphi, S] \equiv \tilde{\bar{A}}_{t}[\varphi, S]-\bar{A}_{t}[\varphi, S]=\left[\bar{D}_{t}, \bar{\theta}\right]-\delta_{S} \bar{A}_{t},
$$

where we have introduced the bar notation to make it clear that the functional dependence of $t$ is only coming through the action.

Globally, two kernels describe the same RG flow between fixed points $i$ and $f$ if their corresponding connections $A_{1}$ and $A_{2}$ produce $t$-ordered lines which are related by

$$
\Phi_{f i}\left[A_{1}\right]=\Omega_{f} \Phi_{f i}\left[A_{2}\right] \Omega_{i}^{-1},
$$

where $\Omega_{i, f}$ are $t$-independent field redefinitions of the fixed points actions at points $i$ and $f$. In perturbation theory, an operator basis is chosen and all field redefinitions turn into finite coupling constant redefinitions. But this is nothing but a change of perturbative scheme, that is of local counterterms, which is also tantamount to a finite redefinition of coupling constants.

As a specific example, the infinitessimal field redefinition

$$
\theta^{\alpha}=\frac{1}{2} \frac{\delta c\left(q^{2}\right)}{q^{2}} \frac{\delta S}{\delta \varphi_{-q}}-\frac{\delta c\left(q^{2}\right)}{c\left(q^{2}\right)} \varphi_{q}
$$

corresponds to changing the cutoff function $c \mapsto c+\delta$ (This may be derived from comparing (12) with some previous observations, $18 \mathrm{~B}$ or directly.) It is thus 
abundantly clear that this change of scheme amounts to a finite redefinition of coupling constants in the continuum theory, with no physical effect.

Another useful version of the exact renormalization group may be obtained by Legendre transformation of $S .10$ The resulting action $\Gamma\left[\varphi^{c}\right]$ has a simple interpretation as an infrared cutoff Legendre effective action and has a corresponding flow

equation.10.11 It is also the effective average action.12 So far, all our analysis has been concerned with the Polchinski form (3) and it would appear that the Legendre flow equation is left out of this party. In general this is true because arbitrary transformations $\theta$ take us to exact renormalization group equations that no longer have the specific form of (3), and thus the direct link to the Legendre effective action is lost. However for the specific transformation (18) the form (3) is left alone: only the cutoff function changes. In this case by utilising the Legendre transform relation, 10 it should be possible to find directly corresponding transformations for $\Gamma$ and $\varphi^{c}$ which leave the the Legendre flow equation invariant up to a change in cutoff function $c \mapsto c+\delta c$.

Finally we would like to emphasise once again the freedom inherent to quantum field theories. Path integrals bring field redefinitions symmetries with deep consequences. The fact that exact renormalization group equations follow from an instance of Schwinger-Dyson type of relations is striking. Their further structure and transformation properties are fully determined by this underlying symmetry.

\section{Acknowledgments}

J.I.L. acknowledges financial support from CICYT (contract AEN98-0431) and CIRIT (contract 1998SGR-00026). T.R.M. acknowledges financial support of PPARC grant GR/K55738. We both acknowledge the financial support of a BCMEC Acciones Integradas grant MDR(A998/99)1799.

\section{References}

1. F. J. Wegner, J. Phys. C7 (1974) 2098.

2. K. G. Wilson and J. Kogut, Phys. Rep. 12C (1974) 75.

3. F. J. Wegner and A. Houghton, Phys. Rev. A8 (1972) 401.

4. J. Polchinski, Nucl. Phys. B231 (1984) 269.

5. T. R. Morris, in The Exact Renormalization Group, Eds Krasnitz et al, World Sci (1999) 1, and hep-th/9810104.

6. J. I. Latorre and T. R. Morris, JHEP 11 (2000) 004, hep-th/0008123.

7. J.-I. Sumi et al, hep-th/0002231.

8. E. K. Riedel, G. R. Golner and K. E. Newman, Ann. Phys. 161 (1985) 178.

9. C. Bagnuls and C. Bervillier, hep-th/0002034.

10. T. R. Morris, Int. J. Mod. Phys. A9 (1994) 2411, hep-ph/9308265.

11. Bonini et al, Nucl. Phys. B409 (1993) 441, hep-th/9301114.

12. C. Wetterich, Phys. Lett. B301 (1993) 90. 